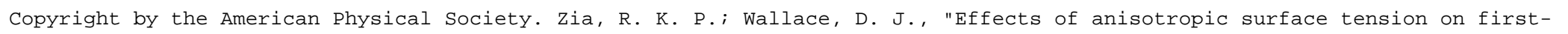
order-transition singularities," Phys. Rev. B 31, 1624 DOI: http://dx.doi.org/10.1103/PhysRevB.31.1624

PHYSICAL REVIEW B

\title{
Effects of anisotropic surface tension on first-order-transition singularities
}

\author{
R. K. P. Zia \\ Physics Department, Virginia Polytechnic Institute and State University, \\ Blacksburg, Virginia 24061 \\ D. J. Wallace \\ Department of Physics, University of Edinburgh, Mayfield Road, \\ Edinburgh EH9 3JZ, Scotland, United Kingdom
}

(Received 8 November 1984)

\begin{abstract}
For systems displaying two-phase coexistence without rotational invariance, anisotropic surface tension and nonspherical droplets are present. To study small fluctuations around such droplets, we construct a natural coordinate system and find the quadratic form. In general, their spectrum differs from the isotropic case and affects the nature of the first-order transition singularities. However, in two bulk dimensions, the spectrum is sufficiently simple that the singularity is universal.
\end{abstract}

Thermodynamic systems whose order parameter has at most a discrete symmetry typically undergo first-order phase transitions between the various discrete phases at low temperatures. Nucleation phenomena involving droplets of one phase embedded in another play an important role at these first-order transitions. A primitive model permitting only spherical droplets ${ }^{1}$ contains an essential singularity. Although this feature persists in more sophisticated calculations, ${ }^{2,3}$ the detailed form of the singularity is modified when fluctuations away from spherical are included. ${ }^{2}$

A specific way to expose the singularity is to consider a uniaxial (Ising) system in a magnetic field $H$. For the stable phase below the critical temperature, say $H>0$, there is a real free-energy function $F(H)$. The existence of the nucleating (or critical) droplet of radius $\propto 1 /|H|$ in the metastable phase implies that the continuation of this function $F(H)$ to $H<0$ has an imaginary part whose form can be calculated for $|H|$ small: ${ }^{2,4}$

$$
\begin{aligned}
& \operatorname{Im} F(\arg H= \pm \pi) \\
& \quad= \pm B|H|^{b} \exp \left\{-A|H|^{-(d-1)}\left[1+O\left(H^{2}\right)\right]\right\} .
\end{aligned}
$$

Here the exponential factor arises from the competition between the volume and surface energies of the droplet. The power $b$ in the prefactor comes from the Gaussian approximation for the fluctuations and is independent ${ }^{4}$ of the details of, e.g., the Landau-Ginzburg potential used to derive (1):

$$
b=\left\{\begin{array}{l}
(3-d) d / 2, \quad 1<d<5, \quad d \neq 3 \\
-7 / 3, \quad d=3
\end{array}\right.
$$

in $d$ (bulk) dimension. Further, higher-order contributions beyond the Gaussian approximation do not appear to change the value of $b$, at least in two dimensions. One predicts then ${ }^{4}$ an essential singularity at first-order transitions which is universal for all (isotropic) Landau-Ginzburg theories. This universality is unrelated to the universality of the critical point; the large length scale, with respect to which microscopic details are irrelevant, is the radius of the nucleating droplet which becomes unboundedly large as coexistence is approached $\left(\mathrm{H} \rightarrow \mathrm{O}^{-}\right)$.

The predicted $H$ dependence in both the exponential and the prefactor of Eq. (1) is in agreement with series expan- sions $^{5,6}$ and Monte Carlo simulation ${ }^{7}$ performed on twodimensional lattice Ising models.

Although the results (1) and (2) capture key features of nucleation, namely, the competition between surface and volume energies, and the effects of surface entropy, there is one important aspect which remains to be incorporated: for temperatures far below $T_{c}$, there are anisotropic effects inherited from the lattice. In particular, the critical droplet is not spherical. The successful comparison mentioned above turns out not to be a sensitive test of anisotropic effects (see below). Phenomenologically this anisotropy may be taken into account through a surface tension $\sigma(\hat{\mathbf{N}})$ which depends on the normal $\hat{\mathbf{N}}$ to the surface. Thus we consider an energy functional

$$
\mathscr{H}[S]=\int_{S} \sigma(\hat{\mathbf{N}}) d a-\int_{\partial V=S}|H| d V
$$

for a droplet bounded by surface $S$. Accordingly, the critical droplet $S_{0}$ is one which extremizes $\mathscr{X}$. The construction relating $\sigma(\hat{\mathbf{N}})$ to the (nonspherical) $S_{0}$ is standard. ${ }^{8}$ For example, in the square lattice Ising model both $\sigma(\hat{\mathbf{N}})$ and $S_{0}$ are known exactly. ${ }^{9}$

The classical contribution of the anisotropy (and of a volume term incorporating the true magnetization) to the amplitude $A$ in (1) is in excellent agreement with the numerical results from the square lattice Ising model, ${ }^{10}$ while the power $-(d-1)$ within the exponential is unaffected by anisotropy. However, the power $b$ in the prefactor will, in general, be sensitive since it arises from the fluctuations around the critical droplet. In particular, for $d=3$ where facets are possible due to positive roughening temperatures, it is conceivable that the critical "droplet" has only facets. An extreme example is the cube, whose fluctuations are elementary and lead to $b=0$ instead of $-7 / 3$ ! Thus, the study of fluctuations about a nonspherical droplet is crucial in determining the (branch point) nature of the singularity of $F$. In this paper, we report first steps in this study. For arbitrary $\sigma$ which is associated with a smooth nonfaceted $S_{0}$, we find the explicit quadratic form governing these fluctuations. Further, we show the surprising result that in $d=2$, the spectrum remains unchanged ${ }^{11}$ for any $T>0$ so that $b=1$ is universal. Some crucial points are indicated here with details to be published elsewhere. ${ }^{12}$

The strategy of the calculation is as follows. Ideally we 
would like to repeat the calculations of Refs. 2 and 4 starting from an anisotropic Landau-Ginzburg model. At present this is not feasible (see later comments). What we can do is to consider the ratio of the partition functions for the anisotropic and isotropic cases. In this ratio, the classical terms have the same structure from the surface-volume competition, as do the Jacobian factors which arise from the $d$ collective coordinates associated with the translation modes. The only difference therefore can come from the ratio of determinants from the two Gaussian integrals, affecting the power $b$. Thus, it suffices to analyze the effects of anisotropy on the spectrum of small oscillations in (3).

Starting from (3), we can write, formally, $S=S_{0}+\delta S$ and expand about $S_{0}$. Since $S_{0}$ is convex, it is possible in principle to specify it by hyperspherical coordinates and to describe $\delta S$ accordingly, but this approach is immensely tedious. Instead, we introduce a set of coordinates which is natural to $\sigma$ and $S_{0}$. Cover the $d$ dimensional Euclidean space by $\left(\theta^{i}, \lambda\right), i=1, \ldots, d-1$. The $\lambda=$ constant surfaces are precisely $S_{0}$ scaled by $\lambda$. Within each surface, a point is labeled by $\theta^{i}$, the angles associated with the normal $\hat{\mathbf{n}}$ to $S_{0}$ at that point. This labeling of points is unique for our class of $\sigma$ 's. Specifically, we start with a dimensionless $\sigma$ (i.e., the physical $\sigma$ in some standard unit $\sigma_{0}$ ) and define a set of functions $\xi^{\mu}(\mu=1, \ldots, d) \operatorname{via}^{13} \xi=\hat{\mathbf{n}} \sigma+\hat{\nabla} \sigma$. Here $\hat{\nabla}$ represents the gradient with respect to $\hat{\mathbf{n}}$. Then the transformation from $(\theta, \lambda)$ to $x^{\mu}$ is given by $x^{\mu}=\lambda \xi^{\mu}\left(\theta^{i}\right)$.

In this coordinate system, $S_{0}$ is simply $\lambda=R \equiv(d-1) \sigma_{0} /$ $|H|$, while $\delta S$ may be described by a scalar function $f$ of $\theta^{i}$ (or $\hat{\mathbf{n}}$ ): $\lambda=R+f\left(\theta^{i}\right)$ with $f<<$. The set of points $\mathbf{x}[f]$ of $S_{0}+\delta S$ are given parametrically by

$$
\mathbf{x}[f]=\left[R+f\left(\theta^{i}\right)\right] \xi\left(\theta^{i}\right) .
$$

From here, the calculation is conceptually straightforward though somewhat involved. We recognize that the surface element $d a$ and the normal $\hat{\mathbf{N}}$ are both functionals of $f$. (Note that $\hat{\mathbf{N}}[0]=\hat{\mathbf{n}}$.) Expanding $\sigma(\hat{\mathbf{N}}[f]) d a[f]$ and $d V$ to second order in $f$ within (3) will yield the quadratic form we seek.

A surface element $d a$ on $\mathbf{x}[f]$ can be represented by the exterior product of all the $d-1$ differentials $x_{i} d \theta^{i}$, where subscripts denote derivatives with respect to $\theta^{i}$. Defining $\mathbf{N}=\mathbf{x}_{1} \times \ldots \times \mathbf{x}_{d-1}$ and $d \Theta \equiv \Pi d \theta^{i}$, we have $d a=|\mathbf{N}| d \Theta$. But $\mathbf{x}_{i}=(R+f) \xi_{i}+f_{i} \xi$ so that $\mathbf{N}$ is linear in $\hat{\nabla} f$. Writing $J$ for the Jacobian in

$$
d^{d} x=J \lambda^{d-1} d \lambda d \Theta
$$

and

$$
\mathbf{F} \equiv \sum_{k}\left(f_{k} / R J\right) \xi_{1} \times \ldots \times \boldsymbol{\xi}_{k-1} \times \boldsymbol{\xi} \times \boldsymbol{\xi}_{k+1} \times \ldots \times \boldsymbol{\xi}_{d-1}
$$

we expand (3) into

$$
\mathscr{H}[f]=\mathscr{H}\left[S_{0}\right]+(f, M f)+O\left(f^{3}\right)
$$

Here

$$
(f, M f) \equiv \frac{1}{2} \sigma_{0} R^{d-1} \int\left[\mathbf{F} Q \mathbf{F}-(d-1) \sigma f^{2} / R^{2}\right] J d \Theta
$$

is the desired quadratic form. $Q$ is the "radius of curvature" tensor $\hat{\nabla} \xi$, which is explicitly ${ }^{13}(1-\hat{\mathbf{n}} \hat{n}) \sigma+\hat{\nabla} \hat{\nabla} \sigma$. Physically, the first term is the "kinetic" one, F being linear in $\partial f / \partial \theta$, while the second is the "mass" term. Note that $M$ has $1+d$ explicit eigenfunctions. One is $f=$ constant, with a negative eigenvalue. This represents the expanding or shrinking mode, against whose perturbations $\mathscr{H}$ is unstable. The others are the $d$ Goldstone modes $(f=\hat{\mathbf{n}} \cdot \epsilon / \sigma)$ representing rigid displacements ${ }^{2,4}$ of $S_{0}$ (by an infinitesimal amount $\boldsymbol{\epsilon}$ ).

As we discussed above, to analyze the effects of anisotropy, we consider the ratio of determinants of $M^{\prime}$, the positive part of $M$ :

$$
\operatorname{det} M^{\prime}[\sigma] / \operatorname{det} M^{\prime}\left[\sigma_{0}\right]=\exp \sum \ln \left\{E_{i}[\sigma] / E_{i}\left[\sigma_{0}\right]\right\},
$$

where $E_{i}>0$ are the eigenvalues. Note that, some of the $E$ 's are undoubtedly degenerate, as in the isotropic case.

This sum is to be cut off when the corresponding eigenstates involve fluctuations on wavelengths smaller than the correlation length; for higher eigenvalues, Eq. (3) is no longer an adequate phenomenological model because of curvature effects or interface structure. On a surface of scale size $R$ the number of contributing modes hence diverges as $R^{d-1} \propto|H|^{-(d-1)}$.

Thus if the ratio of $E$ 's approaches a constant, the amplitude of the $|H|^{-(d-1)}$ term obtained from $\mathscr{H}\left[S_{0}\right]$ would be modified. We are concerned about the possible existence of a logarithmic term from the sum (5) which could contribute to $b$ in (2). Generically, we can expect facets which cannot fluctuate and give rise to infinite $E$ 's (through $Q$ ). Such $E$ 's would be cut off from the sum, leading to changes in (5). Even for our class of $\sigma$ 's, we have not been able to rule out a slow decay (of the ratio of $E$ 's) sufficient to produce a $\ln |H|$ in the sum.

However, much simplification occurs at $d=2$ where faceting is impossible due to zero roughening temperatures. Now $(f, M f)$ is proportional to

$$
\int d \theta\left[\sigma^{2} \dot{f}^{2}-\sigma(\sigma+\ddot{\sigma}) f^{2}\right],
$$

where $\dot{f} \equiv \partial f / \partial \theta$, etc. Letting $\psi \equiv \sigma f$, this is $\int\left[\dot{\psi}^{2}-\psi^{2}\right]$. Thus, the spectrum is identical to the isotropic case and the ratio (5) is precisely unity. So we conclude that the exponent $b$ in the singularity is unaffected by anisotropy in two dimensions.

Apart from the obvious open question concerning the behavior in higher dimensions, several others come immediately to mind. In this paper we restricted ourselves to smooth, nonfaceted equilibrium shapes, so that our coordinate system is nonsingular. But, once the temperature drops below roughening temperatures, facets would appear. Corners and edges can be present even above these temperatures. It would be important to find a way to bypass our restriction.

We have already cited the need to have a clearer understanding of an anisotropic Landau-Ginzburg Hamiltonian which would lead to (3). Preliminary analysis suggests a simple generalization of the "kinetic" term to $\frac{1}{2} \int \sigma^{2}(\hat{\mathbf{N}})(\nabla \phi)^{2}$ where $\hat{\mathbf{N}}(\phi)$ is the unit vector in the direction of $\nabla \phi$. The motivation behind it lies in the interpretation of the "kinetic" term as the energy stored in the gradients of (coarse grained) spin densities. The above generalization represents an energy which depends also on the orientation of these gradients. The Hamiltonian

$$
\int d^{d} x\left[\frac{1}{2} \sigma^{2}(\hat{\mathbf{N}}) \nabla \phi \cdot \nabla \phi+U(\phi)\right],
$$

where $U$ contains the standard double-well potential and magnetic field terms, has the following salient features. (i) 
It is translationally, but not rotationally, invariant with anisotropy clearly incorporated. (ii) The properties of planar interfaces are not modified except for the thickness which is orientation dependent and directly related to $\sigma$. (iii) The highly nonlinear Euler-Lagrange equations associated with (6) reduce to the "radial" equation

$$
\phi^{\prime \prime}+(d-1) \phi^{\prime} / \lambda=\partial U / \partial \phi,
$$

so that the classical solution appropriate to the isotropic case can be used here immediately. Note that $\phi^{\prime}$ is concentrated on the surface of the nonspherical equilibrium shape we studied before. Far from being a closed issue, (6) presents problems of its own, e.g., how does a nonanalytic functional such as $\hat{\mathbf{N}}[\phi]$ come about? Further studies are in progress.

One of us (R.K.P.Z.) thanks the Alexander vonHumboldt Foundation for many forms of support and $\mathrm{H}$. Wagner for the hospitality at the University of Munich. This research is partly supported by Science and Engineering Research Council, United Kingdom Grant No. GR-C-01474.
${ }^{1}$ A. F. Andreev, Zh. Eksp. Teor. Fiz. 45, 2064 (1963) [Sov. Phys. JETP 18, 1415 (1964)].

2J. S. Langer, Ann. Phys. (N.Y.) 41, 108 (1967).

${ }^{3}$ M. E. Fisher, Physics 3, 255 (1967).

${ }^{4}$ N. J. Gunther, D. A. Nicole, and D. J. Wallace, J. Phys. A 13, 1755 (1980). For a review and further references, see D. J. Wallace, in Phase Transitions, proceedings of a Summer Institute, Cargèse, Corsica, France, 1980, edited by M. Levy, J. C. Le Guillou, and J. Zinn-Justin (Plenum, New York, 1982), p. 423.

${ }^{5}$ G. A. Baker, Jr., and D. Kim, J. Phys. A 13, L103 (1980); R. J. Baxter and I. G. Enting, J. Stat. Phys. 21, 103 (1970).

${ }^{6}$ M. J. Lowe and D. J. Wallace, J. Phys. A 13, L381 (1980); see also D. J. Wallace (Ref. 4).

${ }^{7}$ G. Jacucci, A. Perini, and G. Martin, J. Phys. A 16, 369 (1983).

${ }^{8}$ G. Wulff, Z. Kristallog. Mineral. 34, 449 (1901); C. Herring, Phys. Rev. 82, 87 (1951). For a recent review and other references, see R. K. P. Zia, in Statistical and Particle Physics: Common Problems and Techniques, proceedings of the 26th Scottish Universities
Summer School in Physics (SUSSP), 1983, edited by K. C. Bowler and A. J. McKane (SUSSP Publications, Univ. of Edinburgh, 1984).

${ }^{9}$ C. Rottman and M. Wortis, Phys. Rev. B 24, 6274 (1981); J. E. Avron, H. van Beijeren, L. S. Schulman, and R. K. P. Zia, J. Phys. A 15, L81 (1981), and references therein.

${ }^{10}$ C. K. Harris, J. Phys. A 17, L143 (1984); 17, 1767 (1984); see also M. P. A. Fisher, D. S. Fisher, and J. D. Weeks, Phys. Rev. Lett. 48, 368 (1982).

${ }^{11}$ It should be noted that although the equilibrium droplet shape is circular only as $T \rightarrow T_{c}$, it is still nearly circular (Ref. 9) in the square lattice model at $T=\frac{1}{2} T_{c}$. Thus the numerical calculations presently available (Refs. 5-7) for testing (2) cannot be interpreted as strong evidence for the result reported in this paper.

${ }^{12}$ R. K. P. Zia (unpublished).

${ }^{13}$ J. W. Cahn and D. W. Hoffmann, Acta. Metall. 22, 1205 (1974); R. K. P. Zia and J. E. Avron, Phys. Rev. B 25, 2042 (1982). 